\title{
Manipulating Item Proportion and Deception Reveals Crucial Dissociation Between Behavioral, Autonomic, and Neural Indices of Concealed Information
}

\author{
Kristina Suchotzki, ${ }^{1,2 *}$ Bruno Verschuere, ${ }^{1,3,4}$ Judith Peth, $^{2}$ \\ Geert Crombez,' and Matthias Gamer ${ }^{2}$ \\ ${ }^{1}$ Department of Experimental Clinical and Health Psychology, Ghent University, Ghent, \\ Belgium \\ ${ }^{2}$ Department of Systems Neuroscience, University Medical Center Hamburg-Eppendorf, \\ Hamburg, Germany \\ ${ }^{3}$ Department of Clinical Psychology, University of Amsterdam, Amsterdam, The Netherlands \\ ${ }^{4}$ Faculty of Psychology and Neuroscience, Maastricht University, Maastricht, The Netherlands
}

\begin{abstract}
Developed as an alternative to traditional deception detection methods, the concealed information test (CIT) assesses recognition of critical (e.g., crime-relevant) "probes." Most often, recognition has been measured as enhanced skin conductance responses (SCRs) to probes compared to irrelevant foils (CIT effect). More recently, also differentially enlarged reaction times (RTs) and increased neural activity in the bilateral inferior frontal gyrus, the right middle frontal gyrus, and the right temporo-parietal junction have been observed. The aims of the current functional magnetic resonance imaging (fMRI) study were to (1) investigate the boundary conditions of the CIT effects in all three measures and thereby (2) gain more insight into the relative contribution of two mechanisms underlying enhanced responding to concealed information (i.e., orienting versus response inhibition). Therefore, we manipulated the proportion of probe versus irrelevant items, and whether suspects were instructed to actively deny recognition of probe knowledge (i.e., deceive) during the test. Results revealed that whereas overt deception was not necessary for the SCR CIT effect, it was crucial for the RT and the fMRI-based CIT effects. The proportion manipulation enhanced the CIT effect in all three measures. The results indicate that different mental processes might underlie the response pattern in the CIT. While skin conductance responding to concealed information may best be explained by orienting theory, it seems that response inhibition drives RT and blood oxygen level dependent responding to concealed information. Hum Brain Mapp 36:427-439, 2015.
\end{abstract}

\footnotetext{
Additional Supporting Information may be found in the online version of this article.

Contract grant sponsor: ECRP (K.S.); Contract grant number: 09ECRP-025; Contract grant sponsor: FWO; Contract grant number: V410113N; Contract grant sponsor: FWO; Contract grant number: ESF 3G099310; Contract grant sponsor: ECRP (J.P. and M.G.); Contract grant number: 09-ECRP-025; Contract grant sponsor: DFG; Contract grant number: GA 1621/1-1
}

${ }^{*}$ Correspondence to: Kristina Suchotzki; Department of Experimental Clinical and Health Psychology, Ghent University, Henri Dunantlaan 2, B-9000 Ghent, Belgium. E-mail: Kristina.Suchotzki@UGent.be

Received for publication 9 January 2014; Revised 8 September 2014; Accepted 9 September 2014.

DOI: $10.1002 / \mathrm{hbm} .22637$

Published online 3 October 2014 in Wiley Online Library (wileyonlinelibrary.com). 
Key words: concealed information; guilty knowledge; deception; proportion; reaction time, skin conductance; fMRI; response inhibition; orienting; right inferior frontal gyrus

\section{INTRODUCTION}

Developed as an alternative to traditional lie detection methods, the concealed information test [CIT; Lykken, 1959] measures recognition of critical (e.g., crime-related) details. Imagine the theft of a necklace that is only known to the police and the person who reported the theft. To administer a CIT, several questions are developed whose correct answers are only known to a guilty suspect. For instance, one CIT question could be "Do you recognize this item?," presented together with different alternative answers (e.g., a ring, a painting, a necklace, a diamond, and a credit card). Whereas for an innocent suspect all items are equally plausible and, therefore, irrelevant, a guilty suspect is expected to react differently to the correct item (i.e., probe). This differential responding is called the CIT effect (probe minus irrelevant items) and has most often been assessed with measures of the autonomic nervous system. It has been found that probe items compared to irrelevant items elicit increased autonomic responses, with the skin conductance response (SCR) displaying the greatest sensitivity [Ben-Shakhar and Elaad, 2003; Gamer et al., 2008].

More recently, the CIT has been adapted for the assessment of other dependent measures [for a review see Verschuere et al., 2011a], including reaction times [RTs; Seymour et al., 2000] and blood oxygen level dependent activity [BOLD activity; Gamer et al., 2007]. Because these measures require a repeated stimulus presentation, a third item-category has been included into the test to ensure that suspects are paying attention to the item presentation. These "target items" are shown to suspects before administration of the CIT and while suspects are instructed to truthfully admit knowledge (respond "Yes") of the target items, they should deny knowledge (respond "No") of all remaining items (i.e., probes and irrelevant items). With this three-item CIT, probe items compared to irrelevant items were shown to result in prolonged RTs [Seymour and Kerlin, 2008; Verschuere et al., 2010], and an increased neural activity in the bilateral inferior frontal gyrus (IFG), the right middle frontal gyrus (rMFG), and the right temporo-parietal junction [rTPJ; for a recent meta-analysis on the fMRI-based CIT see Gamer, 2011].

Although the SCR is a reliable index of concealed information, several factors have been shown to influence its validity, including item proportion and deception. The higher the proportion of irrelevant items relative to probe items, the greater the SCR CIT effect [Ben-Shakhar, 1977; Ben-Shakhar et al., 1982; Lieblich et al., 1970]. Results were less homogeneous regarding the effect of overt deception.
Some researchers found that when guilty suspects were required to actively deceive during the test (i.e., deny probe knowledge by responding "No" to every item) the SCR CIT effect was larger compared to when suspects were not required to actively deceive [i.e., give no responses or respond "Yes" to every item; see e.g., Elaad and Ben-Shakhar, 1989; Furedy and Ben-Shakhar, 1991; Gustafson and Orne, 1965; Horneman and O'Gorman, 1985]. Others, however, could not replicate these findings [Kugelmass et al., 1967; Verschuere et al., 2009a], and in two meta-analyses on moderators of the SCR CIT effect, the factor deception failed to reach significance [Ben-Shakhar and Elaad, 2003; Meijer et al., 2014]. With regard to the validity of RT and fMRI-based concealed information measures, there is no research so far on the role of item proportion, but first research on the role of overt deception. The majority of research using RTs indicates that when participants are not asked to actively deny probe knowledge, the RT CIT effect disappears [Matsuda et al., 2009; Meijer et al., 2007; Verschuere et al., 2009b, but see Labkovsky and Rosenfeld, 2012; Rosenfeld and Labkovsky, 2010; Rosenfeld et al., 2008 for opposing findings]. The only study using fMRI found that the neural CIT effect was still significant without active denial of probe knowledge [Gamer et al., 2012].

With regard to the mechanisms underlying the CIT, the main research focus has been on the Orienting Response [OR, Sokolov, 1963]. The OR is a conglomerate of behavioral and physiological responses that is elicited by rare, novel or personally significant stimuli. The OR can explain the proportion effect and the majority of other findings with the CIT. However, some research findings regarding the autonomic responses to concealed information cannot be accounted for by mere orienting [for a review, see Verschuere and Ben-Shakhar, 2011]. Importantly, the differentially enhanced RTs and neural activity in the rIFG for probe items led to the idea that another mechanism might contribute to the CIT effect: Response inhibition. Response inhibition is defined as an executive function that allows one to intentionally inhibit a dominant, automatic, or prepotent response [Miyake et al., 2000]. It was reasoned that the denial of probe knowledge causes a conflict for guilty suspects between the truthful "Yes" response and the tobe-given deceptive "No" response, which can only be solved by inhibiting the former response [Seymour and Schumacher, 2009; Verschuere and De Houwer, 2011].

Support for the role of response inhibition comes from the aforementioned findings that RT differences between probe and irrelevant items disappear once overt deception, and therefore, overt response conflict, is removed from the 
paradigm [Matsuda et al., 2009; Meijer et al., 2007; Verschuere et al., 2009b]. Furthermore, the rIFG, whose activity is reliably modulated by the CIT, is considered an area crucially involved in inhibitory processes, as it is consistently activated during traditional response inhibition tasks [e.g., Aron et al., 2004, 2014; Derrfuss et al., 2005; Garavan et al., 1999; Kelly et al., 2004; Liddle et al., 2001; Picton et al., 2007; Rubia et al., 2003; van Boxtel et al., 2001]. However, Gamer et al. [2012] obtained significantly enhanced rIFG activity for probe compared to irrelevant items in the absence of deception and overt response conflicts. The authors, therefore, hypothesized that the enhanced rIFG activity could result from the infrequent presentation of the probes, since enhanced rIFG activity has also been linked to the detection of rare events in a stream of more frequently occurring events [Bledowski et al., 2004; Chatham et al., 2012; Downar et al., 2000; Hampshire et al., 2007; Herron et al., 2004; Strange et al., 2000]. This interpretation suggests that the rIFG plays a more general role for attentional processes, which is more in line with the traditional orienting account.

In sum, scientific evidence reveals that concealed information can be indexed by behavioral, autonomic, and neural signatures, but it remains unclear which factors influence their respective validity and which mechanisms underlie the response pattern in different measures. From the available literature it seems that the SCR CIT effect is augmented particularly by a proportion effect, and less by overt deception, and that it is largely determined by the OR. In contrast, RT and fMRI measures may be more strongly determined by overt deception and may, therefore, rely at least partly on response inhibition. The aim of this study was twofold. First, by manipulating item proportion and deception within each examinee we examined the boundary conditions of the CIT. More specifically, we wanted to investigate which manipulations may enhance CIT effects in different measures of concealed information (RTs, SCRs, and BOLD responses), and which conditions may even be necessary for CIT effects to occur. Second, determining the degree to which these manipulations affect CIT effects in different measures should allow us to gain more insight into the relative contributions of orienting versus response inhibition. Whereas the orienting account predicts that proportion will impact most heavily on responding, the response inhibition account predicts stronger effects when participants are instructed to overtly deceive than when they may reply truthfully.

\section{METHOD}

\section{Participants}

Thirty-two healthy, right-handed volunteers (15 women, 17 men) participated for a monetary reward. The study was approved by the ethical committee of the University Medical Center Hamburg-Eppendorf and conformed to the principles expressed in the Declaration of Helsinki. All participants provided written informed consent. The mean age in the sample was 25.41 years $(S D=4.26$ years).

\section{Experimental Design}

In our design, all participants conducted a two-stage mock-crime. Deception was manipulated within subjects by instructing participants to admit knowledge of half of the mock-theft related probes, and to deny knowledge of the other half of the mock-theft related probes. Proportion was also manipulated within subjects by varying the probe versus irrelevant item proportion, with either 1 probe to 1 irrelevant item or 1 probe to 4 irrelevant items. This resulted in a fully crossed $2 \times 2$ design with the CIT effect (i.e., the difference score of probe minus irrelevant items) as dependent variable and Deception (admit vs. deny) and Proportion (1:1 vs. 1:4) as within-subject factors, manipulated in four different CIT blocks. Although target items were used during the task to ensure participants' attention, they were not included in any of our analyses as they were not informative for our hypotheses.

\section{Procedure}

Participants were welcomed by a first experimenter and were informed that they were participating in a lie detection study. They were explained that they would receive an instruction to commit two mock thefts and that afterwards, a second, naïve experimenter would aim to detect whether they were actually involved in the crimes or not. All participants were instructed to enter a storage room and to (1) look on the left side of the room for a key with a little car on the keychain. With that key they should open a red money box that was hidden in a striped colorful bag and contained a 50 Euro note. Additionally, participants were instructed to (2) go to the right side of the room and move an empty water crate to access a storage container. In that container they should find a brown envelope containing a yellow sheet with a password ("mickey.100"). They should use a red text marker to note the password on a post-it. With the money and the password participants should return to the instruction room. All relevant details that were used for the construction of the CIT are printed in italics above.

After completion of the two mock thefts, the first experimenter informed the participants that a second naive experimenter would conduct a lie detection test concerning their involvement in both thefts. Participants were told that the second experimenter has enough evidence that they were guilty of the first theft, but that she did not know whether they were also guilty of the second theft. Participants were explained that truthfully admitting the first crime, while convincingly denying the second crime would increase their chance of being considered innocent of the second theft. The order in which both mock crimes had to be executed and the instruction which crime had to 
TABLE I. Trial type examples for all four experimental conditions (blocks)

\begin{tabular}{|c|c|c|c|c|c|}
\hline $\begin{array}{l}\text { Deception } \\
\text { Condition }\end{array}$ & $\begin{array}{l}\text { Proportion } \\
\text { Condition }\end{array}$ & $\begin{array}{l}\text { Stimulus } \\
\text { type }\end{array}$ & $\begin{array}{l}\text { Example Stimuli } \\
\text { (pictures) }\end{array}$ & $\begin{array}{l}\text { Number } \\
\text { of trials }\end{array}$ & $\begin{array}{l}\text { Correct } \\
\text { Response }\end{array}$ \\
\hline \multirow[t]{6}{*}{ Admit } & \multirow[t]{3}{*}{$1: 1$} & Irrelevant & Laptop & 40 & No \\
\hline & & Probe & 50 Euro note & 40 & Yes \\
\hline & & Target & $\mathrm{CD}$ & 40 & Yes \\
\hline & \multirow[t]{3}{*}{$1: 4$} & Irrelevant & $\begin{array}{l}\text { Rucksack, Leather } \\
\text { bag, Plastic bag, } \\
\text { Safe }\end{array}$ & 160 & No \\
\hline & & Probe & Striped colorful bag & 40 & Yes \\
\hline & & Target & Suitcase & 40 & Yes \\
\hline \multirow[t]{6}{*}{ Deny } & \multirow[t]{3}{*}{$1: 1$} & Irrelevant & Yellow text marker & 40 & No \\
\hline & & Probe & Red text marker & 40 & No \\
\hline & & Target & Green fine liner & 40 & Yes \\
\hline & \multirow[t]{3}{*}{$1: 4$} & Irrelevant & $\begin{array}{l}\text { Trashcan, Toilet } \\
\text { paper, Printer } \\
\text { Paper, Printer Ink }\end{array}$ & 160 & No \\
\hline & & Probe & Water crate & 40 & No \\
\hline & & Target & Toolbox & 40 & Yes \\
\hline
\end{tabular}

Note: The examples listed correspond to a participant that had to admit the money theft, but deny the password theft.

be admitted and which had to be denied were counterbalanced between participants. To increase participants' motivation, they were promised 30 Euro for an overall accurate and fast performance across all four experimental blocks, combined with a convincing admittance and denial of the respective mock thefts (three participants were paid this extra reward based on their pattern of behavioral responses ${ }^{1}$ ).

Thereafter, participants were sent to another building to meet the second experimenter and take the test. Participants received detailed task instructions and practiced the task outside the MRI scanner (with autobiographical questions and stimuli unrelated to the thefts). Finally, before completion of the CIT in the MRI scanner, participants studied and memorized the target items until they could remember all of them correctly.

\section{CIT paradigm}

The CIT in the MRI scanner was presented on a translucent screen using a video projector, which subjects viewed via a mirror mounted to the head coil. During the entire task the question "Do you recognize this object?" was presented in the upper middle part of the screen. Left and right button presses were used to indicate "yes" and "no" responses and reminder labels were presented left and right below the question. Participants were asked to press

\footnotetext{
${ }^{1}$ The extra reward was paid when participants achieved (1) more than $95 \%$ correct responses (2) a mean RT of under $700 \mathrm{~ms}$, and (3) a negative mean RT CIT effect in the admit and the deny condition, respectively.
}

the correct button as accurately and quickly as possible using the index and middle finger of their right hand. The association of response labels to the index and middle finger was counterbalanced across subjects. As stimuli, pictures of eight theft-related probe items, eight target items and 32 irrelevant items were used (see Supporting Information Fig. 1). The assignment of probes (and associated irrelevant and target items) to experimental blocks was randomized within each Deception condition (admit vs. deny). Furthermore, when a 1:1 proportion of probe and irrelevant items was used, the respective irrelevant item was determined randomly out of all four possible irrelevant items. Each block began with an irrelevant buffer item that was excluded from the data-analyses. After that, stimuli were presented in randomized order, with an inter stimulus interval varying randomly (drawn from a beta distribution) between 1 and 8 seconds $(M=3$ s). To encourage fast responding, a $1000 \mathrm{~ms}$, response deadline was used, after which the stimulus disappeared and the words "Too slow" were presented.

Table I provides an illustration of the four different experimental blocks, the responses that had to be given on different trials and the number of trials per item and block. Block order was counterbalanced, with the only restriction that the two corresponding Deception conditions were always paired together.

After the CIT and outside the MR scanner, participants completed a brief explicit memory test for the theft items. All participants remembered all probe items. Finally, participants performed a standard version of the Stop-Signal task and a keyboard Stroop task [Logan, 1994; Stroop, 1935; retrieved from http://www.millisecond.com/download/library/] to get independent estimates of the 
individual response inhibition ability. Since we did not find systematic correlations between these two tasks and the CIT effects in the RT, SCR, and fMRI measures, we do not report results of these tasks. These data can be provided on request.

\section{Data Acquisition}

Stimulus presentation and recording of error rates and RTs were performed with the software Presentation (Neurobehavioral Systems, Albany, CA). SCRs were recorded using a constant voltage system (MP100, Biopac Systems, Goleta, CA) with $\mathrm{Ag} / \mathrm{AgCl}$ electrodes placed on the thenar and hypothenar eminences of the left hand. Responses were amplified and digitized at $200 \mathrm{~Hz}$.

Functional imaging was performed on a 3-Tesla wholebody MR scanner (Magnetom Trio, Siemens, Erlangen, Germany), equipped with a 32-channel head coil. Forty transverse slices (slice thickness $2 \mathrm{~mm}$; $1 \mathrm{~mm}$ gap) were acquired in each volume covering the whole brain. A T2*sensitive gradient echo-planar imaging (EPI) sequence was used (repetition time $[\mathrm{TR}]=2260 \mathrm{~ms}$; echo time $[\mathrm{TE}]=26$ $\mathrm{ms}$; flip angle $=80^{\circ} ; \mathrm{FOV}=220 \times 220 \mathrm{~mm}$; in-plane resolution $2 \times 2 \mathrm{~mm}$ ). Additionally, isotropic high-resolution (1 $\times 1 \times 1 \mathrm{~mm}^{3}$ ) structural images were recorded using a T1-weighted coronal-oriented MPRAGE sequence with 240 slices.

\section{Data Preprocessing and Analysis}

\section{Behavioral responses}

Buffer items, incorrect responses $(1.88 \%)$ and responses exceeding the response deadline (i.e., longer than $1000 \mathrm{~ms}$; $1.48 \%$ ) were excluded from the RT analysis. RTs for irrelevant and probe items were averaged for each of the four conditions, and the CIT effect $\left(M_{\text {probes }}-M_{\text {irrelevants }}\right)$ was computed for each condition. To analyze the influence of the experimental manipulations on the behavioral data, we calculated $2 \times 2$ MANOVAs on error rate and RT differences with Deception (admit vs. deny) and Proportion (1:1 vs. 1:4) as within-subject factors. As a measure of effect size the standardized mean difference $d$ was calculated [Cohen's $d$ for paired data; Dunlap et al., 1996; Morris and DeShon, 2002], with 0.20, 0.50, and 0.80 as thresholds for "small," "moderate," and "large" effects [Cohen, 1988].

\section{Skin conductance responses}

Because of the response overlap resulting from the short inter stimulus interval in our design, it was necessary to first decompose the recorded skin conductance into tonic and phasic components, using an individually fitted template of a discrete SCR for each participant [Lim et al., 1997]. In a first step, the algorithm, that was implemented using R (http://www.r-project.org), generated a template to match the individual SCR morphology. This template was optimized by minimizing the squared difference between the measured electrodermal data and the modeled response. In a second step, this SCR template was fitted to the whole skin conductance tracing of the respective participant. Additional SCRs were added when the model fit related to its complexity, determined using a Bayesian information criterion, increased. The procedure resulted in a set of SCRs for each electrodermal recording that best resembled the measured data. Subsequently, SCRs that were elicited by the stimuli were identified by searching for responses with an onset between 1 and $3 \mathrm{~s}$ after stimulus onset. The amplitudes of these responses were logtransformed using the natural logarithm to reduce the skewness of the distribution.

Error trials and trials exceeding the response deadline were excluded from the SCR analysis. SCR amplitudes for irrelevant and probe items were averaged for each of the four conditions, and the CIT effect $\left(M_{\text {probes }}-M_{\text {irrelevants }}\right)$ was computed for each condition. Finally, we calculated a $2 \times 2$ MANOVA on these amplitude differences with Deception (admit vs. deny) and Proportion (1:1 vs. 1:4) as within-subject factors. As a measure of effect size the standardized mean difference $d$ was calculated [Cohen's $d$ for paired data; Dunlap et al., 1996; Morris and DeShon, 2002], with $0.20,0.50$, and 0.80 as thresholds for "small," "moderate," and "large" effects [Cohen, 1988].

\section{BOLD responses}

Imaging data were preprocessed and analyzed with Statistical Parametric Mapping (SPM8, Wellcome Department of Imaging Neuroscience, London). The first four volumes of each session were discarded to allow for signal equilibration. Data of each participant were slice-time corrected and realignment with unwarping was performed for each session. The high-resolution T1 image was coregistered with the mean EPI image and segmented using the unified segmentation approach as implemented in the "New Segment" routine in SPM8. During this latter preprocessing step, tissue-class images for gray and white matter were generated and used within the DARTEL toolbox to create structural templates across subjects as well as individual flow fields [Ashburner, 2007]. These flow fields were then used to spatially normalize the EPI images into MNI space. Images were saved with a spatial resolution of $2 \times 2 \times 2 \mathrm{~mm}^{3}$, and smoothing was accomplished using a 6-mm full-width at half maximum (FWHM) isotropic Gaussian kernel. Finally, images were high-pass filtered at $128 \mathrm{~s}$, and an autoregressive AR(1) model was used to account for serial correlations in fMRI time series.

To analyze BOLD responses as a function of the experimental manipulations, we modeled probes and irrelevant items as separate regressors in each of the four experimental conditions and convolved them with the hemodynamic response function. Target items, buffer items, error trials, and trials exceeding the response deadline were modeled separately as regressors of no interest. Simple contrast maps 
(probe items vs. irrelevant items) were generated for each subject and each condition individually. A flexible factorial model (two-way within subjects ANOVA, including a subject factor) was used to test for main and interaction effects of Deception (deny vs. admit) and Proportion (1:4 vs. 1:1) on the activation pattern. For all analyses, a family-wise error rate (FWE) threshold of $P<0.05$, corrected for predefined regions of interests (ROIs), was used. These regions were defined by $12 \mathrm{~mm}$ spheres centered on the MNI coordinates from a meta-analysis on the fMRI-based CIT [Gamer, 2011: rIFG 39/23/-10, rMFG 35/44/23, rTPJ: 60/-48/30, IIFG: $-44 / 19 /-1]$. Activations outside these ROIs were reported if they survived a whole brain correction for multiple comparisons using an FWE threshold of $P<0.05$ and a cluster threshold of $k>20$ voxels. To illustrate the pattern of activity and to identify the conditions contributing to the interaction of Deception $\times$ Proportion, we conducted post hoc analyses on the contrast estimates in selected ROIs. These values were derived from the peak voxel of a given contrast using the toolbox rfxplot [Gläscher, 2009]. For illustration purposes, voxels are displayed as statistical maps thresholded at $P<0.001$, uncorrected, cluster extent $k>40$ voxels, which are overlaid on the mean, skull-stripped structural image from all participants. The respective activations are reported in $x / y / z$ coordinates in MNI space.

\section{Effect sizes CIT effect}

To obtain an indication of test performance, the standardized mean difference $d$ was calculated for the CIT effects in all four conditions $\left(d=M_{\text {probe-irrelevant }} /{ } S D_{\text {probe-irrelevant }}\right)$, separately for each of the three dependent measures: RTs, SCRs, and BOLD activity in all four ROIs $5 \mathrm{~mm}$ spheres at the coordinates reported by Gamer, 2011] as well as the mean activity of all four ROIs. As a rule of thumb, Cohen [1988] proposed 0.20, 0.50, and 0.80 as thresholds for "small," "moderate," and "large" effects.

\section{RESULTS}

\section{Behavioral responses}

Mean RTs are shown in Figure 1A. The $2 \times 2$ MANOVA on RTs revealed a significant main effect of Deception, $F(1$, $31)=30.17, P<0.001, d=0.97,95 \%$ CI $[0.55,1.39]$, with a significant and large CIT effect in the deny condition, $t(31)=5.72, P<0.001, d=1.01,95 \%$ CI $[0.58,1.44]$, and a significantly reversed medium CIT effect in the admit condition, $t(31)=-2.91, P<0.01, d=-0.51,95 \%$ CI $[-0.88,-0.14]$. Furthermore, there was a significant main effect of Proportion, $F(1,31)=52.21, P<0.001, d=1.28,95 \%$ CI $[0.81,1.74]$, with a significant large CIT effect in the $1: 4$ condition, $t(31)=5.79, P<0.001, d=1.03,95 \%$ CI $[0.60,1.46]$, and a significantly reversed medium CIT effect in the 1:1 condition, $t(31)=-3.46, P<0.01, d=-0.61,95 \%$ CI $[-0.99,-0.23]$. The interaction of Deception $\times$ Proportion showed a tendency towards significance, $F(1,31)=3.27, P=0.08, d=0.32,95 \%$
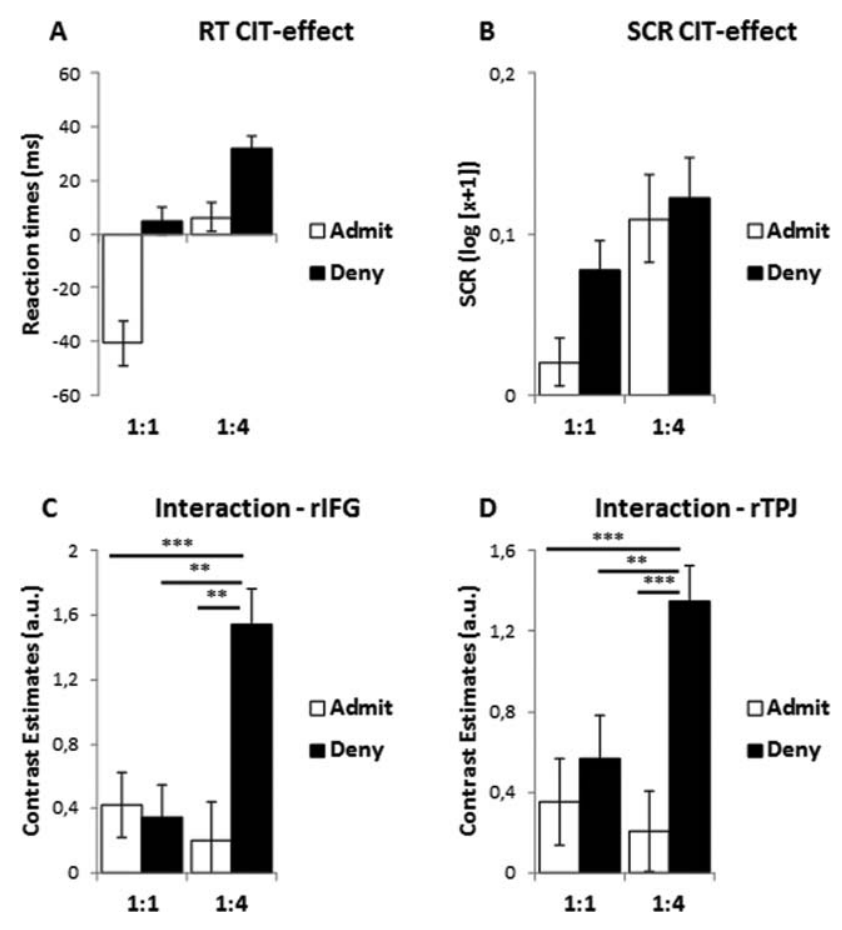

Figure I.

Mean CIT effects (probe minus irrelevant items) in RTs (A) and SCRs (B) for all four conditions. Contrast estimates for CIT effects in the peak voxels of the two ROls that showed a significant interaction effect between Deception and Proportion: the right IFG (C) and the right temporo-parietal junction (D). Error bars indicate standard errors of the mean. $x=$ SCR in $\mu$ S. a.u. $=$ arbitrary unit. $* * * P<0.001$, $* * P<0.01$.

CI $[-0.04,0.67]$. The $2 \times 2$ MANOVA on error rates did not reveal any significant effects, all $F^{\prime} \mathrm{s}<2.34, P^{\prime} \mathrm{s}>0.14$.

\section{Skin Conductance Responses}

Mean SCRs are shown in Figure 1B. The $2 \times 2$ MANOVA on the SCRs revealed a significant main effect of Deception, $F(1,31)=5.47, P<0.05, d=0.41,95 \%$ CI [0.05, 0.77], with a larger CIT effect in the deny condition, $t(31)=5.82, P<0.001, d=1.03,95 \%$ CI $[0.60,1.45]$, compared to the admit condition, $t(31)=3.79, P<0.01, d=0.67$, $95 \%$ CI $[0.29,1.05]$. Furthermore, there was a significant main effect of Proportion, $F(1,31)=16.42, P<0.001$, $d=0.72,95 \%$ CI $[0.33,1.11]$, with a larger CIT effect in the 1:4 condition, $t(31)=5.25, P<0.001, d=0.93,95 \%$ CI $[0.51$, 1.32], compared to the $1: 1$ condition, $t(31)=4.39, P<0.001$, $d=0.77,95 \%$ CI $[0.38,1.17]$. The interaction was not significant, $F(1,31)=1.12, P=0.30, d=0.23,95 \%$ CI $[-0.12,0.59]$.

\section{BOLD Responses}

In all four ROIs, we found a main effect of Deception, with a significantly larger BOLD CIT effect for the deny 
TABLE II. Brain regions showing significant main effects of Deception and Proportion and regions showing a significant interaction of Deception $\times$ Proportion

\begin{tabular}{|c|c|c|c|c|c|c|c|}
\hline \multirow[b]{2}{*}{ Effect } & \multirow{2}{*}{$\begin{array}{l}\text { Regions contained } \\
\text { within cluster }\end{array}$} & \multicolumn{3}{|c|}{$\begin{array}{l}\text { Peak voxel (MNI } \\
\text { coordinates) }\end{array}$} & \multirow{2}{*}{$\begin{array}{l}\text { Cluster size } \\
\text { (voxels) }\end{array}$} & \multirow[b]{2}{*}{ Z } & \multirow{2}{*}{$\begin{array}{l}\text { Corrected } \\
P \text { (FWE) }\end{array}$} \\
\hline & & $x$ & $y$ & $z$ & & & \\
\hline \multirow[t]{4}{*}{ Main effect Deception (Deny $>$ Admit) } & Right inferior frontal gyrus & 44 & 24 & -4 & 165 & 4.49 & 0.001 \\
\hline & Right middle frontal gyrus & 34 & 52 & 24 & 59 & 4.24 & 0.004 \\
\hline & Right temporo-parietal junction & 62 & -46 & 28 & 654 & 5.75 & 0.000 \\
\hline & Left inferior frontal gyrus & -42 & 16 & 0 & 155 & 4.48 & 0.002 \\
\hline \multirow[t]{6}{*}{ Main effect Proportion $(1: 4>1: 1)$} & Right inferior frontal gyrus & 48 & 22 & -6 & 186 & 4.77 & 0.000 \\
\hline & Right middle frontal gyrus & 34 & 50 & 22 & 256 & 4.77 & 0.000 \\
\hline & Right temporo-parietal junction & 58 & -50 & 20 & 108 & 4.02 & 0.009 \\
\hline & Left inferior frontal gyrus & -36 & 16 & 6 & 123 & 4.40 & 0.002 \\
\hline & ${ }^{\mathrm{a}}$ Left postcentral gyrus & -44 & -34 & 52 & 170 & 6.11 & 0.000 \\
\hline & ${ }^{\mathrm{a}}$ Left precentral gyrus & -36 & -6 & 62 & 86 & 5.81 & 0.000 \\
\hline \multirow{2}{*}{$\begin{array}{l}\text { Interaction effect Deception } \\
\quad \times \text { Proportion }\end{array}$} & Right inferior frontal gyrus & 46 & 24 & -10 & 34 & 4.14 & 0.006 \\
\hline & Right temporo-parietal junction & 52 & -42 & 36 & 153 & 4.22 & 0.004 \\
\hline
\end{tabular}

Note: Only the peak voxel of each cluster is reported. The spatial cluster extent was $k \geq 20$ voxels and a small volume correction (sphere of $12 \mathrm{~mm}$ around previously defined ROIs) with a family-wise error rate of $P_{\mathrm{FWE}}<0.05$ was used to correct for multiple comparisons.

${ }^{a}$ Additional areas that were significant on a whole brain level (spatial extent $k \geq 20$ voxels and $P_{\mathrm{FWE}}<0.05$ ).

condition compared to the admit condition, and a main effect of Proportion with a significantly larger BOLD CIT effect for the 1:4 condition compared to the 1:1 condition (see Table II, Fig. 2).

In the rIFG and the rTPJ, we additionally found a significant interaction of both factors (see Fig. 2). Posthoc $t$ tests revealed that in both regions these interactions were mainly driven by the BOLD CIT effect in the deny 1:4 condition, which differed significantly from the CIT effects in all other three conditions (see Figs. 1C,D). As can be seen in Figure 3, there was a partial overlap of the activations of the main and interaction effects in the rIFG and the rTPJ.

\section{Effect Sizes CIT Effect}

The effect sizes of the different CIT effects varied considerably between RT, SCR, and fMRI measures and the four experimental conditions (see Table III). Reducing the number of irrelevant relative to probe items or asking participants to admit instead of deny probe knowledge substantially reduced effect sizes of the RT and BOLD CIT effects. This reduction was less strong for the effect sizes of the SCR CIT effects.

Effect sizes and confidence intervals further indicated that the RT CIT effect was largest in the deny 1:4 condition, and significantly exceeded zero only in that condition. Also the SCR CIT effect was largest in the deny 1:4 condition, but it remained large and significant in the deny $1: 1$ and the admit 1:4 conditions. All BOLD CIT effects were largest in the deny 1:4 condition and differed significantly from zero in that condition. None of the BOLD CIT effects in the admit 1:1 condition reached sig- nificance. In the deny 1:1 condition, CIT effects were significant when BOLD activity was averaged across all four ROIs and in two of the four ROIs. These effects were of medium to large size. In the admit 1:4 condition, CIT effects were significant when BOLD activity was averaged across all four ROIs and in one of the four ROIs. These effects were of small to medium size.

\section{DISCUSSION}

This study pursued two aims. Our first aim was to clarify which factors contribute to RT, skin conductance and fMRI-based CIT effects. We therefore focused on two theoretically important factors: Deception and Proportion. Specifically, we (1) instructed participants to admit knowledge of half of the mock crime-related probe items and deny knowledge of the other half, and (2) manipulated the proportion of probe versus irrelevant items (1:1 vs. 1:4). Moreover, studying the effects of both manipulations should allow us to draw further conclusions on the contributions of orienting and response inhibition to the CIT effects in different measures. Deception and Proportion affected the CIT effects in all three dependent measures, albeit to a different extent. This finding revealed important dissociations between RT, SCR, and fMRI-based concealed information measures.

For the RT measure, the effects were dramatic. As is clear from an inspection of Figure 1A, the CIT effect was robust in the deny 1:4 condition, yet significantly reversed in the admit 1:1 condition. That the CIT effect was not just absent in the admit 1:1 condition, but reversed may be related to the proportion of "yes" versus "no" responses in that particular condition. Only the irrelevant 


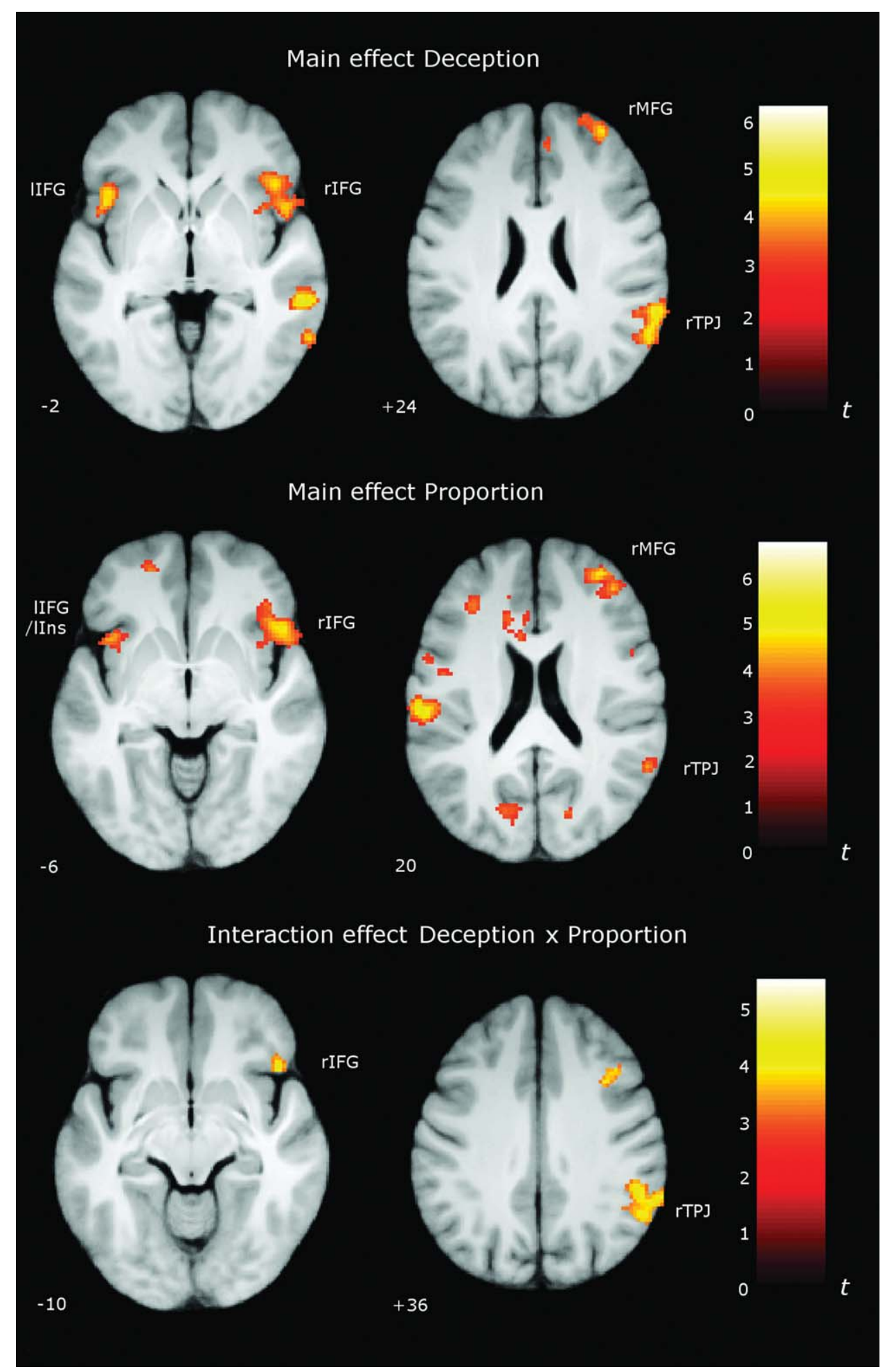

Figure 2.

Brain regions showing significant main effects of deception and vated voxels are displayed as statistical maps thresholded at proportion and brain regions showing a significant interaction of Deception $\times$ Proportion. Regions are displayed on axial slices (MNI z-coordinates are given) of a mean, skull-stripped structural image from all participants. For illustration purposes, acti$P<0.001$, uncorrected, cluster extent $k>40$ voxels. rIFG $=$ right inferior frontal gyrus; IIFG = left inferior frontal gyrus; rMFG = right middle frontal gyrus; rTPJ = right temporoparietal junction; Ilns = left insula. 


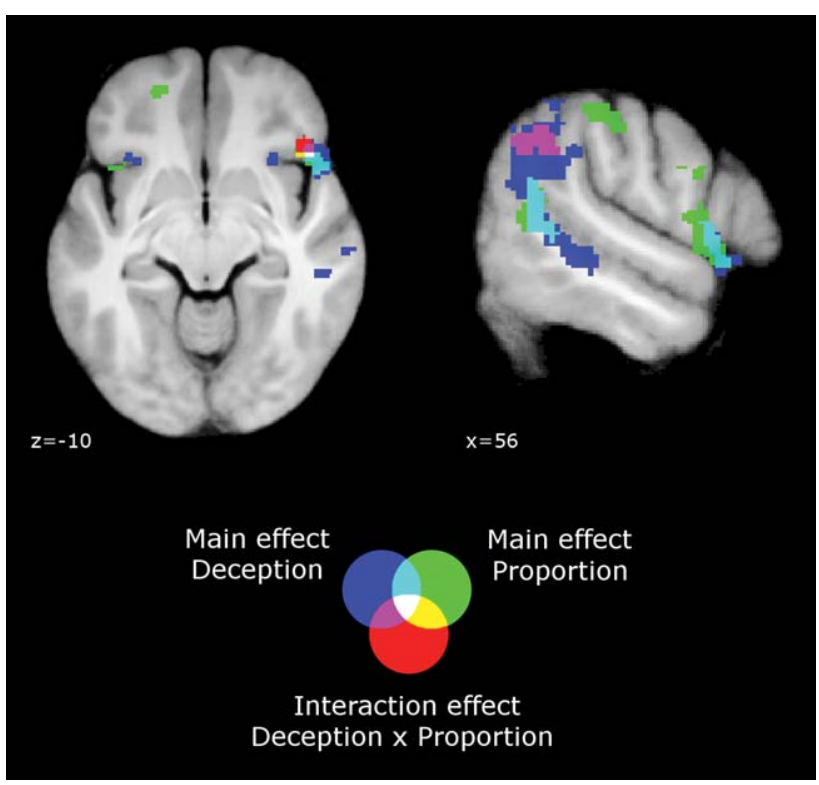

Figure 3.

Overlay of the significant main and interaction effects. Regions are displayed on axial and sagittal slices (MNI z- or $x$-coordinates are given) of a mean, skull-stripped structural image from all participants. For illustration purposes, activated voxels are displayed as statistical maps thresholded at $P<0.00$ I, uncorrected, cluster extent $k>40$ voxels.

stimuli required a "no" response, whereas both the probe and the target stimuli required a "yes" response. The higher proportion of "yes" responses might have facilitated "yes" responses compared to "no" responses [Pashler and Baylis, 1991], resulting in a reversed CIT effect. Our results are in line with previous research [Matsuda et al., 2009; Meijer et al., 2007; Verschuere et al., 2009b], and indicate that to obtain an RT CIT effect, deception is not just a contributing, but rather a necessary factor. The fact that the RT CIT effect only reached significance in the deny 1:4 condition indicates that also a larger proportion of irrelevant compared to probe items may be necessary for the RT CIT effect to occur. This result is in contrast with results obtained in many deception paradigms. Although it has for instance in the Sheffield lie task [Spence et al., 2001] been found that a higher proportion of irrelevant (truth) trials enhances differential RT effects to probe (lie) trials [Verschuere et al., 2011b; Van Bockstaele et al., 2012], robust RT results are usually also obtained with a 1:1 proportion [e.g., Debey et al., 2012; Fullam et al., 2009; Spence et al., 2001, 2004, 2008]. As there are no previous studies manipulating item proportion in the RT CIT, further research seems warranted before any strong conclusions on the role of item proportion can be drawn.

For the SCR measure, both Deception and Proportion contributed to the CIT effect, with no significant interaction. The significant proportion effect corresponds with

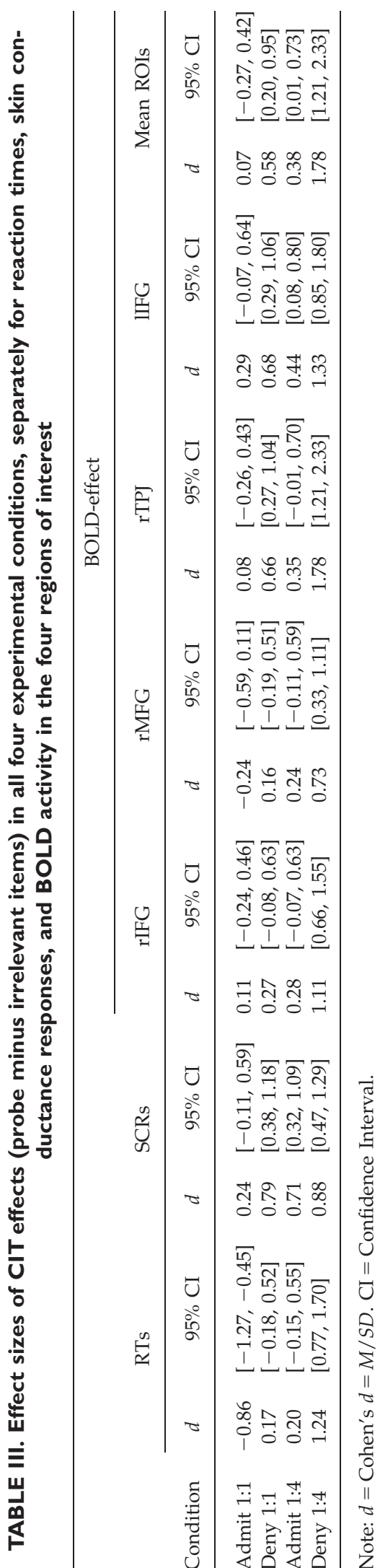


the findings that increasing the set size of irrelevant items also leads to an increased detection rate in the traditional two-item SCR CIT [Ben-Shakhar, 1977; Ben-Shakhar et al., 1982; Lieblich et al., 1970]. The significant deception effect further corresponds with studies reporting that active denial of probe knowledge enhanced the SCR CIT effect in the two-item CIT [Elaad and Ben-Shakhar, 1989; Furedy and Ben-Shakhar, 1991; Gustafson and Orne, 1965; Horneman and O'Gorman, 1985], but contrasts with studies failing to find such an effect [e.g., Kugelmass et al., 1967; Verschuere et al., 2009a]. However, although both Proportion and Deception increased the size of the SCR CIT effect, neither appeared necessary, as evidenced by the significant CIT effects in the deny $1: 1$ and the admit 1:4 conditions. This result is consistent with the finding that although there exist numerous moderators of the SCR CIT effect, it can already be obtained under minimal conditions [BenShakhar and Elaad, 2003; Meijer et al., 2014].

The BOLD CIT effect appeared robust in all four ROIs in the deny 1:4 condition. But whereas CIT effects lacked significance in three of the four ROIs in both admit conditions, CIT effects in the rTPJ and the IIFG as well for the average across all four ROIs were significant and even of medium to large size in the deny 1:1 condition. This strongly indicates that, like the RT CIT effect, the fMRI-based CIT effect critically depends on Deception. This result seems in conflict with the findings of Gamer et al. [2012], who found enhanced rIFG activity for probes as compared to irrelevant CIT items in the absence of apparent response conflict. There is, however, a crucial difference between the instructions used in both studies. Although the CIT paradigm by Gamer et al. [2012] did not contain any overt response conflict (participants simply had to indicate seeing every item with the same button press), the overall goal for the participants was still to hide certain information (i.e., deception). In contrast, in our design, participants were explicitly told that this particular crime knowledge was already known to the experimenters. Note also that despite the fact that participants still had to deceive in the Gamer et al. [2012] study, the RT CIT effect disappeared. This may be explained by the fact that the behavioral responses in the Gamer et al. [2012] study were not contingent on stimulus content. Thereby, participants could respond before stimulus content may have been sufficiently processed [Suchotzki et al., 2013].

In addition to the results obtained for predefined ROIs, we also found a modulation of neural activity by Proportion in the pre- and postcentral gyrus of the left hemisphere. These regions overlap with the somatosensory representation and motor control of the right hand [e.g., Bear et al., 2006], which was used to deliver behavioral responses. Therefore, this result might simply be related to differences in the overall frequency of button presses, but it may also reflect enhanced attentional saliency of behavioral responses to probe items when the number of irrelevant items increases.

Taken together, our results indicate an interesting dissociation between RT, skin conductance and fMRI-based measures of concealed information. Whereas our propor- tion manipulation enhanced the effect in all three measures, overt deception was crucial for the RT and the fMRIbased CIT effect, but not for the SCR CIT effect. This latter finding also fits with data of a recent study by Matsuda et al. [2013]. In that study, participants were instructed to hide knowledge of one of two probes, stating that one was "discovered" by the experimenter before the test started. Although participants in their study did not overtly admit or deny any knowledge during the CIT but simply pressed the same response button for every item, these authors still found a dissociation between several autonomic and different event-related potential (ERP) measures. Whereas CIT effects in SCRs, heart rate, cutaneous blood flow, and the N2 and P300 ERP components were present for both probes, CIT effects in the respiration measure and the late positive potential were only significant when probe knowledge needed to be concealed.

Knowledge on how deception and proportion affect the different CIT effects also provides further information on their underlying theoretical mechanisms (i.e., orienting versus response inhibition). Our finding that deception constitutes a necessary condition for the RT CIT effect supports the idea that task characteristics are crucial to obtain reliable RT effects [Verschuere and De Houwer, 2011]. More specifically, a manipulation of conflict between the truthful "Yes" response and the to-be-given deceptive "No" response seems crucial for a successful RT CIT, and points to the role of response inhibition in this task. Regarding our SCR results, there are two possible interpretations. The first is that orienting as well as response inhibition processes contribute independently to the SCR CIT effect. The second, and more economical interpretation, is that the orienting account can incorporate the effects of both the deception and the proportion manipulation. As orienting increases with stimulus salience, the deception manipulation may have increased probe salience, thus boosting the OR. Although response inhibition may be at play, the most parsimonious explanation for the SCR CIT effect then is to interpret the SCR CIT effect within OR theory. For the fMRI-based CIT effects (except for the IIFG), the CIT effect completely vanished in the admit conditions. These results strongly indicate that deception may be a necessary condition for the fMRI-based CIT effects and thereby also point towards response inhibition as their crucial underlying mechanism. This further corresponds with findings that the rIFG is crucially involved in traditional response inhibition tasks [Aron et al., 2004, 2014; Derrfuss et al., 2005; Garavan et al., 1999; Kelly et al., 2004; Liddle et al., 2001; Picton et al., 2007; Rubia et al., 2003; van Boxtel et al., 2001], and that also in our experiment, Deception significantly modulated rIFG activity. Our results for the rIFG and the IIFG differ slightly. The IIFG was the only ROI in which a significant CIT effect was observed in the admit 1:4 condition. In general, CIT effects were more pronounced in the IIFG compared to the rIFG. The stronger enhancement of the IIFG CIT effect by Deception (compared to Proportion) corresponds with findings 
of bilateral IFG activation in response inhibition tasks and supports the idea that also the IIFG may essentially contribute to response inhibition processes [Swick et al., 2008]. Yet, interestingly, not only Deception but also Proportion significantly modulated neural activity in all four ROIs in our experiment and both factors even interacted in the rIFG and the rTPJ. According to Aron et al. [2014], such a modulation of rIFG activity by proportion may best be explained by increased inhibitory demands for salient and infrequent stimuli, which would indicate that attentional effects strengthen primarily response inhibition-based CIT effects. However, note that our results also fit with findings and claims of other authors proposing that rIFG, rTPG, and rMFG activity may not be specific for one mental process, but rather reflect an interplay of multiple processes in complex cognitive tasks [Corbetta et al., 2008; Corbetta and Shulman, 2002; Dodds et al., 2011; Duncan and Owen, 2000; Hampshire et al., 2010]. Within that view, the interaction effects found in the rIFG and the rTPJ would probably rather be explained more generally by a strong increase (beyond a purely additive effect) of task complexity in the deny 1:4 condition.

Our results have clear practical implications. The CIT effects varied substantially between conditions and measures, ranging from reversed CIT effects to very large effects. All three measures produced the largest effects in the deny 1:4 condition, which therefore seems the most optimal for applied purposes. Comparing effect sizes in this condition between different measures revealed large effects for all measures (except for the BOLD-activity in the rMFG). The effect in the SCR measure was smaller compared to the effects in the RT and fMRI measures, and also compared to the SCR effect size found in two previous meta-analyses [ $d=1.55$; Ben-Shakhar and Elaad, 2003; Meijer et al., 2014]. The most likely explanation for this discrepancy is that our short intertrial interval and the large number of stimulus repetitions may have been suboptimal for the SCR recording. The effect sizes of the RT as well as the majority of the BOLD CIT effects were very large, with the largest effects in the rTPJ and the mean activity of all four ROIs. However, as indicated by the substantial overlap of the confidence intervals, future research is needed to determine whether the differences between our effect sizes reflect genuine differences between these measures. Our findings also provide suggestions on how to further enhance the CIT effect in the different measures. RT and fMRI-based CIT effects could be maximized by interfering with response inhibition capacities. In contrast, for the SCR CIT effect, it may be more promising to concentrate on task manipulations that enhance the OR. Combining the assessment of two measures that tap into different mechanisms could also further increase the diagnostic power of the CIT.

Our study also has limitations. First, although several aspects of our study contribute to its external validity (i.e., using a mock crime procedure; a cover story; a different experimenter during the mock crime phase; an incentive for appearing unknowledgeable), the external validity remains limited (e.g., university students, instructed deception). Second, we did not include an innocent control group to compare specificity between measures. However, it is important to note that in contrast to other deception detection techniques [Lykken, 1974], the specificity of the CIT only depends on the test structure (i.e., the number of questions and items). Given a properly constructed test (i.e., all items are equally plausible for an innocent suspect), specificity estimates should therefore be comparable between measures when similar techniques are used to analyze response strengths for probes and irrelevant items. Third, it is important to acknowledge that it is unlikely that our manipulations of proportion and deception only tapped into orienting or response inhibition processes, respectively. Deception might also have influenced the salience of the probe items and motivational processes in general. More specifically, the condition in which participants had to deny probe knowledge might have led to an increased motivation to conceal probe knowledge and thereby also to an increased salience of these probe items. To avoid this, we explicitly stated in our instructions that chances to avoid "conviction" and gain an extra financial reward depended on both a convincing admission as well as a convincing denial of knowledge. It is also possible that our proportion manipulation not only influenced orienting, but also attentional processes in general (e.g., by depleting attentional resources in the 1:4 condition).

To sum up, our results show that whereas overt deception was not necessary for the SCR CIT effect to occur, it was crucial for the RT and the fMRI-based CIT effects. The proportion manipulation enlarged the CIT effect in all three measures. These results indicate that different mental processes might underlie the response pattern in the CIT. While skin conductance responding to concealed information may best be explained by orienting theory, it seems that response inhibition drives RT and BOLD responding to concealed information.

\section{ACKNOWLEDGMENT}

We thank Marie Bartholomäus and Gina Gutsche for their help with the recruitment of participants and data collection and Katrin Müller, Kathrin Wendt, and Timo Krämer for their help with MR scanning. We thank Bram van Bockstaele for his comments on an earlier version of the manuscript.

\section{REFERENCES}

Aron AR, Robbins TW, Poldrack RA (2004): Inhibition and the right inferior frontal cortex. Trends Cogn Sci 8:170-177.

Aron AR, Robbins TW, Poldrack RA (2014): Inhibition and the right inferior frontal cortex: One decade on. Trends Cogn Sci 18:177-185.

Ashburner J (2007): A fast diffeomorphic image registration algorithm. NeuroImage 38:95-113. 
Bear MF, Connors BW, Paradiso MA (2006): Neuroscience: Exploring the Brain. Philadelphia: Lippincott.

Ben-Shakhar G (1977): A further study of the dichotomization theory in detection of information. Psychophysiology 14:408403.

Ben-Shakhar G, Elaad E (2003): The validity of psychophysiological detection of information with the Guilty Knowledge Test: A meta-analytic review. J Appl Psychol 88:131-151.

Ben-Shakhar G, Lieblich I, Kugelmass S (1982): Interactive effects of stimulus probability and significance on the skin conductance response. Psychophysiology 19:112-114.

Bledowski C, Prvulovic D, Goebel R, Zanella FE, Linden DE (2004): Attentional systems in target and distractor processing: A combined ERP and fMRI study. NeuroImage 22:530-540.

Chatham CH, Claus ED, Kim A, Curran T, Banich MT, Munakata Y (2012): Cognitive control reflects context monitoring, not motoric stopping, in response inhibition. PLoS One 7:e31546.

Cohen J (1988): Statistical Power Analysis for the Behavioural Sciences. Hillsdale: Lawrence Erlbaum.

Corbetta M, Shulman GL (2002): Control of goal-directed and stimulus-driven attention in the brain. Nature reviews. Nat Rev Neurosci 3:201-215.

Corbetta M, Patel G, Shulman GL (2008): The reorienting system of the human brain: from environment to theory of mind. Neuron 58:306-324.

Debey E, Verschuere B, Crombez G (2012): Lying and executive control: An experimental investigation using ego depletion and goal neglect. Acta Psychol 140:133-141.

Derrfuss J, Brass M, Neumann J, von Cramon DY (2005): Involvement of the inferior frontal junction in cognitive control: Metaanalyses of switching and Stroop studies. Hum Brain Mapp 25:22-34.

Dodds CM, Morein-Zamir S, Robbins TW (2011): Dissociating inhibition, attention, and response control in the frontoparietal network using functional magnetic resonance imaging. Cereb Cortex 21:1155-1165.

Downar J, Crawley AP, Mikulis DJ, Davis KD (2000): A multimodal cortical network for the detection of changes in the sensory environment. Nat Neurosci 3:277-283.

Duncan J, Owen AM (2000): Common regions of the human frontal lobe recruited by diverse cognitive demands. Trends Neurosci 23:475-483.

Dunlap WP, Cortina JM, Vaslow JB, Burke MJ (1996): Meta-analysis of experiments with matched groups or repeated measures designs. Psychol Methods 1:170-177.

Elaad E, Ben-Shakhar G (1989): Effects of motivation and verbalresponse type on psychophysiological detection of information. Psychophysiology 26:442-451.

Fullam RS, McKie S, Dolan MC (2009): Psychopathic traits and deception: Functional magnetic resonance imaging study. Br J Psychiatry 194:229-235.

Furedy JJ, Ben-Shakhar G (1991): The roles of deception, intention to deceive, and motivation to avoid detection in the psychophysiological detection of guilty knowledge. Psychophysiology 28:163-171.

Gamer M (2011): Detection of deception and concealed information using neuroimaging techniques. In: Verschuere B, BenShakhar G, Meijer E, editors. Memory Detection: Theory and Application of the Concealed Information Test. Cambrige: Cambridge University Press. pp 90-114.

Gamer M, Bauermann T, Stoeter P, Vossel G (2007): Covariations among fMRI, skin conductance, and behavioral data during processing of concealed information. Hum Brain Mapp 28: $1287-1301$.

Gamer M, Verschuere B, Crombez G, Vossel G (2008): Combining physiological measures in the detection of concealed information. Physiol Behav 95:333-340.

Gamer M, Klimecki O, Bauermann T, Stoeter P, Vossel G (2012): fMRI-activation patterns in the detection of concealed information rely on memory-related effects. Soc Cogn Affect Neurosci 7:506-515.

Garavan H, Ross TJ, Stein EA (1999): Right hemispheric dominance of inhibitory control: An event-related functional MRI study. Proc Natl Acad Sci USA 96:8301-8306.

Gläscher J (2009): Visualization of group inference data in functional neuroimaging. Neuroinformatics 7:73-82.

Gustafson LA, Orne MT (1965): The effects of verbal responses on the laboratory detection of deception. Psychophysiology 2:1013.

Hampshire A, Duncan J, Owen AM (2007): Selective tuning of the blood oxygenation level-dependent response during simple target detection dissociates human frontoparietal subregions. J Neurosci 27:6219-6223.

Hampshire A, Chamberlain SR, Monti MM, Duncan J, Owen AM (2010): The role of the right inferior frontal gyrus: Inhibition and attentional control. NeuroImage 50:1313-19.

Herron JE, Henson RN, Rugg MD (2004): Probability effects on the neural correlates of retrieval success: An fMRI study. NeuroImage 21:302-310.

Horneman CJ, O'Gorman JG (1985): Detectability in the card test as a function of the subject's verbal response. Psychophysiology 22:330-333.

Kelly AM, Hester R, Murphy K, Javitt DC, Foxe JJ, Garavan H (2004): Prefrontal-subcortical dissociations underlying inhibitory control revealed by event-related fMRI. Eur J Neurosci 19: 3105-3112.

Kugelmass S, Lieblich I, Bergman Z (1967): The role of "lying" in psychophysiological detection. Psychophysiology 3:312-315.

Labkovsky E, Rosenfeld JP (2012): The P300-based, complex trial protocol for concealed information detection resists any number of sequential countermeasures against up to five irrelevant stimuli. Appl Psychophysiol Biofeedback 37:1-10.

Liddle PF, Kiehl KA, Smith AM (2001): Event-related fMRI study of response inhibition. Hum Brain Mapp 12:100-109.

Lieblich I, Kugelmass S, Ben-Shakhar G (1970): Efficiency of GSR detection of information as a function of stimulus set size. Psychophysiology 6:601-608.

Lim CL, Rennie C, Barry RJ, Bahramali H, Lazzaro I, Manor B, Gordon E (1997): Decomposing skin conductance into tonic and phasic components. Int J Psychophysiol 25:97-109.

Logan GD (1994): On the ability to inhibit thought and action: A user's guide to the stop signal paradigm. In: Carr DDTH, editor. Inhibitory Processes in Attention, Memory, and Language. San Diego: Academic Press. pp 189-239.

Lykken DT (1959): The Gsr in the detection of guilt. J Appl Psychol 43:385-388.

Lykken DT (1974): Psychology and the lie detector industry. Am Psychol 29:725-739.

Matsuda I, Nittono H, Hirota A, Ogawa T, Takasawa N (2009): Event-related brain potentials during the standard autonomicbased concealed information test. Int J Psychophysiol 74:58-68.

Matsuda I, Nittono H, Ogawa T (2013): Identifying concealmentrelated responses in the concealed information test. Psychophysiology 50:617-626. 
Meijer EH, Smulders FT, Merckelbach HL, Wolf AG (2007): The P300 is sensitive to concealed face recognition. Int J Psychophysiol 66:231-237.

Meijer EH, Klein Selle N, Elber L, Ben-Shakhar G (2014): Memory detection with the concealed information test: A meta analysis of skin conductance, respiration, heart rate, and p300 data. Psychophysiology 51:879-904.

Miyake A, Friedman NP, Emerson MJ, Witzki AH, Howerter A, Wager TD (2000): The unity and diversity of executive functions and their contributions to complex "frontal lobe" tasks: A latent variable analysis. Cognitive Psychol 41:49-100.

Morris SB, DeShon RP (2002): Combining effect size estimates in meta-analysis with repeated measures and independentgroups designs. Psychol Methods 7:105-125.

Pashler H, Baylis G (1991): Procedural learning .1. locus of practice effects in speeded choice tasks. J Exp Psychol Learn 17:2032.

Picton TW, Stuss DT, Alexander MP, Shallice T, Binns MA, Gillingham S (2007): Effects of focal frontal lesions on response inhibition. Cereb Cortex 17:826-838.

Rosenfeld JP, Labkovsky E (2010): New P300-based protocol to detect concealed information: Resistance to mental countermeasures against only half the irrelevant stimuli and a possible ERP indicator of countermeasures. Psychophysiology 47:10021010.

Rosenfeld JP, Labkovsky E, Winograd M, Lui MA, Vandenboom C, Chedid E (2008): The Complex Trial Protocol (CTP): A new, countermeasure-resistant, accurate, P300-based method for detection of concealed information. Psychophysiology 45:906919.

Rubia K, Smith AB, Brammer MJ, Taylor E (2003): Right inferior prefrontal cortex mediates response inhibition while mesial prefrontal cortex is responsible for error detection. NeuroImage 20:351-358.

Spence SA, Farrow TFD, Herford AE, Wilkinson ID, Zheng Y, Woodruff PWR (2001): Behavioural and functional anatomical correlates of deception in humans. Neuroreport 12: $2849-2853$.

Spence SA, Hunter MD, Farrow TFD, Green RD, Leung DH, Hughes CJ, Ganesan V (2004): A cognitive neurobiological account of deception: Evidence from functional neuroimaging. Philos Trans R Soc B 359:1755-1762.

Spence SA, Kaylor-Hughes CJ, Brook ML, Lankappa ST, Wilkinson ID (2008): 'Munchausen's syndrome by proxy' or a 'miscarriage of justice'? An initial application of functional neuroimaging to the question of guilt versus innocence. Eur Psychiatry 23:309-314.

Seymour TL, Kerlin JR (2008): Successful detection of verbal and visual concealed knowledge using an RT-based paradigm. Appl Cogn Psychol 22:475-490.
Seymour TL, Schumacher EH (2009): Electromyographic evidence for response conflict in the exclude recognition task. Cogn Affect Behav Neurosci 9:71-82.

Seymour TL, Seifert CM, Shafto MG, Mosmann AL (2000): Using response time measures to assess "guilty knowledge". J Appl Psychol 85:30-37.

Sokolov EN (1963): Perception and the Conditioned Reflex. New York: Macmillan.

Strange BA, Henson RN, Friston KJ, Dolan RJ (2000): Brain mechanisms for detecting perceptual, semantic, and emotional deviance. NeuroImage 12:425-433.

Stroop JR (1935): Studies of interference in serial verbal reactions. J Exp Psychol 18:643-662.

Suchotzki K, Verschuere B, Crombez G, De Houwer J (2013): Reaction time measures in deception research: Comparing the effects of irrelevant and relevant stimulus-response compatibility. Acta Psychol 144: 224-231.

Swick D, Ashley V, Turken U (2008): Left inferior frontal gyrus is critical for response inhibition. BMC Neurosci 9:102.

Van Bockstaele B, Verschuere B, Moens T, Suchotzki K, Debey E, Spruyt A (2012): Learning to lie: Effects of practice on the cognitive cost of lying. Front Psychol 3:526.

van Boxtel GJM, van der Molen MW, Jennings JR, Brunia CHM (2001): A psychophsiological analysis of inhibitory control in the stop-signal paradigm. Biol Psychol 58:229-262.

Verschuere B, Ben-Shakhar G (2011): Theory of the concealed information test. In: Verschuere B, Ben-Shakhar G, Meijer E, editors. Memory Detection: Theory and Application of the Concealed Information Test. Cambridge: Cambride University Press. pp 128-148.

Verschuere B, De Houwer J (2011): Detecting concealed information in less than a second: Response latency-based measures. In: Verschuere B, Ben-Shakhar G, Meijer E, editors. Memory Detection: Theory and Application of the Concealed Information Test. Cambridge: Cambridge University Press. pp 46-63.

Verschuere B, Crombez G, Smolders L, De Clercq A (2009a): Differentiating orienting and defensive responses to concealed information: The role of verbalization. Appl Psychophysiol Biofeedback 34:237-244.

Verschuere B, Rosenfeld JP, Winograd MR, Labkovsky E, Wiersema R (2009b): The role of deception in P300 memory detection. Legal Criminol Psychol 14:253-262.

Verschuere B, Crombez G, Degrootte T, Rosseel Y (2010): Detecting concealed information with RTs: Validity and comparison with the polygraph. Appl Cognitive Psychol 24:991-1002.

Verschuere B, Ben-Shakhar G, Meijer E (2011a): Memory Detection: Theory and Application of the Concealed Information Test. Cambridge: Cambridge University Press.

Verschuere B, Spruyt A, Meijer EH, Otgaar H (2011b): The ease of lying. Conscious Cogn 20:908-911. 